tuber-about 90 per cent of the whole-can be used as food. It is said that tips yield as well as whole tubers and that their produce is less subject to disease. In 1942, in the U.S.S.R., 250,000 acres were sown with potato tips, and it is anticipated that ten times this area will be thus sown in 1943. This would mean an additional 8 or 9 million tons of potatoes in 1943 without having appreciably reduced the quantity available for food and industry in 1942 .

\section{Methods of Clearing Derelict Land}

THE clearing and reclamation of derelict land has become an urgent problem on many farms in Britain to-day. In view of the recent developments in the mechanization of British agriculture, the new edition of Bulletin No. 101, "Hedge and Tree-Stump Clearing", by T. Swarbrick, recently published by the Ministry of Agriculture (price 6d.), should prove of great help to farmers faced with probably unfamiliar operations. The methods recommended fall into four groups : hand methods, the use of power, chemical agents and explosives. The means selected depends upon a variety of circumstances. Much can be done with timber jacks and monkey winches, especially when isolated trees are the chief problem. If hedges also need removing and no tractor is available, the use of a monkey winch in conjunction with explosives is suggested, for horses can then pull out the small stuff. As regards power methods, the track-laying type of tractor is undoubtedly most suitable for land clearing, but it is essential that full use be made of the dead weight of the tractor, its drawbar pull and the leverage exerted by making a high hitch on the tree and a luw hitch on the tractor. Other forms of power such as steam tackle, gyro-tillers and bulldozers can also do most valuable work, if the size of the job justifies the use of expensive equipment. Explosives, particularly gelignite, offer a simple means of removing tree stumps and hedges; full details are given in the bulletin as to how they should be employed. Farmers requiring further information or help are recommended to get in touch with the machinery sections of the county war agricultural committees, who are in possession of the necessary tractors and employ full-time machinery officers to organize the equipment.

\section{School Science Teaching}

THE recent issue of the School Science Review (No. 92, Nov. 1942) contains an interesting article, "School Science Teaching after the War", by Mr. E. T. Harris. Mr. Harris points out that science teaching before the War was greatly affected by economic and social conditions which, in some cases, created a hostile attitude. But now, due to its contribution to the war effort, science is valued and appreciated. The changed social conditions likely to prevail after the War should give impetus to the modern movement to stress the applications of science, but the principles should not be omitted. ". . . Science is a social phenomenon, and is only to be understood in relation to the human society in which it has developed and is developing. Its principles and its applications are closely interrelated aspects of the same social phenomenon, and they must be studied in conjunction." In discussing how this principle may be applied in teaching, Mr. Harris cites the introduction of general science and the greater attention which biology is receiving nowadays (see Nature, 149, 456; April 25, 1942). There are two aspects to the study of science, (1) its historical and logical development and its social uses, and (2) its application to the pupil's life-his home, body, food, etc. Later in the science course should come a broadening of these early ideas, so that science is regarded as a struggle of mankind to master Nature for knowledge, power, and freedom.

\section{A New Universal Bevel Protractor}

Messrs. E. R. WATtS \& SoN, LTD., 123 Camberwell Road, London, S.E.5, have designed and introduced a new type of bevel gauge which should prove a most valuable tool in the engineering workshop for the convenient and accurate measurement of angles. The instrument consists essentially of two straightedges hinged together by means of the protractor head and capable of being set at any desired angle. The principal feature of this bevel gauge is the circular scale, which is divided accurately on a glass annulus mounted inside the head. The graduations on this scale are read by means of a high-power magnifier attached to the head and giving a wide field of view. The scale is most conveniently observed by looking through the eyepiece when the instrument is held in front of a source of artificial light, and under these conditions angles can be measured to within one twelfth of a degree. The straight-edges or blades are made of hardened steel and one of them is capable of sliding, an arrangement which greatly extends the range of the instrument, and two sizes of blade are provided-6 in. and $12 \mathrm{in.}$ The sliding blade is secured by an eccentric operated by a lever extending from the centre. When fitted with the short sliding blade, the dimensions of the gauge, closed, are $6 \frac{1}{2}$ in. $\times 2 \frac{8}{8}$ in. $\times 1 \frac{8}{4}$ in. and its accuracy in measurement is 5 minutes of angle. The blades are secured in angular position by means of a knurled ring concentric with and surrounding the protractor head so that, in all respects, this tool lends itself to rapid and convenient operation.

\section{The Royal Observatory, Cape of Good Hope}

ThE 1941 report of H.M. Astronomer at the Cape, though showing only too clearly the impact of the War on South African astronomy, contains much of interest. The reversible transit circle has been fairly fully employed in making 7,234 transit observa. tions, including fifty-seven of the moon, which were undertaken in view of the fact that lunar observations have perforce been dropped from the restricted programmes of many European observatories. With the Victoria telescope the stellar parallax programme has been continued, 2,642 plates having been secured during the year. A new determination of the parallax of Proxima Centauri, the star closest to the sun, gives $0.763^{\prime \prime} \pm 0.007^{\prime \prime}$, in good agreement with the previously accepted figure of $0.762^{\prime \prime} \pm 0 \cdot 005^{\prime \prime}$; this should be compared with the value $0.756^{\prime \prime} \pm 0.007^{\prime \prime}$ for $\alpha$ Centauri. It is interesting to note that during recent years the number of plates used for a parallax determination has increased to thirty, taken over three years or more: this change is fully in accord with the experience gained in the cloudier weather (but better seeing) at Greenwich.

The photoheliograph record of the sun's disc has suffered somewhat from the shortage of fine-grain plates, but a record, either on lantern plates or faster emulsions, was obtained on 311 days. Observations of occultations by the moon indicate a correction of $0 \cdot 77^{\prime \prime}$ to its ephemeris longitude, which is of course based on Brown's Tables. This correction is expected to reach zero in 1943 . Cometary 
observations included the photographing of Comet $1941 c$ (de Kock), which reached the second magnitude, and of Comet Cunningham, which did not live up to expectations and only just reached naked-eye brightness. The weather at the Cape over the year was cloudier than usual, rainfall being 40 per cent above normal, as the result of considerable falls on a large number of days rather than excessive falls on a few days. Substantial observing losses through cloud were recorded in the programmes of the Victoria telescope and of the photoheliograph, but in spite of the weather the increased efforts of a wardepleted staff actually raised the number of observations made with the transit circle.

\section{A New Telephone Set for Partially Deaf Persons}

An article by A. Herckmans (Bell Lab. Rec., 21, No. 2; October 1942) describes a new amplifying set which recently has been developed for use with the regular telephone set to assist the hard of hearing, and which permits all the added equipment except a small $4 \frac{1}{2}$-volt battery to be incorporated in the base of the telephone. The gain of the amplifier is adjusted by turning one of the switchhook plungers, and the amplifier can be disconnected by a second switch controlled by this same plunger. The switchhook plunger at the right of the set gives gain control and it may be turned to any one of three positions to give low, medium or high gain. Vertical motion of the plunger removes the amplifier from the circuit entirely. The very small amplifier unit consists of a granular-carbon microphone the diaphragm of which is actuated by a bipolar receiver element using permanent magnets. A soft rubber mounting prevents it from picking up vibrations from the surface on which the set is resting. When the amplifier is in use its receiver element is connected in place of the regular handset receiver, while its transmitter element is connected to the handset receiver in series with a $4 \frac{1}{2}$-volt battery which may be placed in any convenient location and connected to the set by two wires. With this arrangement the handset receiver is operated by the amplified speech from the transmitter element of the amplifier.

To prevent 'singing', the amount of gain around the closed path from the receiver to the transmitter through the air path and then back to the receiver through the induction coil must be less than the total loss. A maximum of $25 \mathrm{db}$. gain is provided by the amplifier used and 'singing' will not occur in normal use. A person is not conscious of impairment of hearing for ordinary room conversations until his loss is about $25 \mathrm{db}$., and only about 5 per cent of the population would be conscious of the need for a hearing aid. This percentage would be smaller for telephone conversations because the average speech level received is approximately $10 \mathrm{db}$. higher than that of normal direct conversation. With the additional $25 \mathrm{db}$. provided by the new set, persons with as much as $60 \mathrm{db}$. loss will receive adequate volume. Since only 0.5 per cent have a loss greater than this, the new set provides the help needed for about 90 per cent of those conscious of hearing impairment and would undoubtedly give considerable assistance to many with losses in excess of this.

\section{Application of Electricity in Mine Pumping}

G. B. Alvey and N. Tetlow, in a paper on this subject read before the Institution of Electrical Engineers on December 10, give a good deal of useful information upon electrically driven mine-drainage pumps, which may be of the centrifugal or of the positive displacement reciprocating type. Since the characteristics of the driven pump have a considerable bearing on the choice of the most suitable driving motor, the first portion of the paper deals with operating and design characteristics of the two types of pump. The remainder shows how, by a study of these operating characteristics, the most suitable type of driving motor may be chosen.

\section{Control of Venereal Diseases in Peru}

LAws concerning the medical care of patients suffering from venereal diseases in Peru have recently been promulgated by the President (J.Amer. Med. Assoc., October 17). A national department dealing with these diseases has recently been established with its headquarters in Lima. Treatment during the contagious stage is obligatory. If the patients are minors or mentally deficient, the parents are made responsible for their treatment. Drugs for the treatment of venereal disease are now sold only on medical prescriptions, and the sale of specific medicine by unauthorized persons and treatment by correspondence are prohibited. Wet nurses must have a certificate of health, which is given free of charge in the dispensaries and hospitals. Five per cent of all the beds in public hospitals are to be reserved for venereal diseases.

\section{Edinburgh Geological Society: Clough Memorial Medal and Fund}

THe Clough Memorial Medal for the years 19411942 has been presented to Mr. James L. Begg, of Mount Vernon, Glasgow, for his outstanding contributions to Scottish palæontology. He has worked for many years on the Ordovician rocks of Southern Scotland and has discovered more than a hundred new species of trilobites, mollusca, brachiopods, and other organisms, many of which belong to new genera. The majority of the specimens have been obtained from the classical sections of the Girvan region. Mr. Begg, who is a past president of the Geological Society of Glasgow, has also served as its honorary treasurer for the past twenty-five years.

The Clough Memorial Fund was instituted in 1935 for the purpose of encouraging geological research in Scotland and the north of England. The north of England is defined as comprising the counties of Northumberland, Cumberland, Durham, Westmorland, and Yorkshire. Under the terms of the administration of the fund a sum of approximately $£ 30$ is available annually. Applications for grants are invited for the period April 1, 1943-March 31, 1944. These should be sent to the Secretary, Clough Research Fund Committee, "Edinburgh Geological Society, Synod Hall, Castle Terrace, Edinburgh.

\section{Greetings from Russian Physiologists}

Prow. A. V. HruL, joint secretary of the Royal Society and foreign secretary of the Physiological Society, has received a cable of New Year greetings from the All-Union and Moscow Societies of Physiologists, Pharmacologists and Biochemists, expressing their confidence in complete victory during 1943 for the democratic countries. In his reply, Prof. Hill said he looked forward to joining with thein in holding the Congress of Physiologists deferred from 194I in Great Britain during 1944. 